\title{
Polarimetry with azimuthally polarized light
}

\author{
Juan Carlos González de Sande ${ }^{\mathrm{a}, *}$, Gemma Piquero $^{\mathrm{b}}$, Massimo Santarsiero ${ }^{\mathrm{c}}$ \\ ${ }^{a}$ ETSIS de Telecomunicación, Universidad Politécnica de Madrid, Campus Sur 28031, Madrid, Spain \\ ${ }^{b}$ Departamento de Óptica, Universidad Complutense de Madrid, Ciudad Universitaria 28040 Madrid, \\ Spain \\ ${ }^{c}$ Dipartimento di Ingegneria, Università Roma Tre, Via V. Volterra 62, 00146 Rome, Italy
}

\begin{abstract}
Nonuniformly polarized light can be used for Mueller polarimetry of homogeneous linear samples. In this work, a set up based on using azimuthally polarized input light and a modified commercial light polarimeter is proposed and developed. With this set up, a Mueller submatrix of a sample can be obtained by measuring the Stokes parameters at only three different positions across the output beam section. Symmetry constraints for linear deterministic samples allow the complete Mueller matrix to be deduced for this kind of specimens. The experimental results obtained for phase plates and for a linear polarizer confirm the validity of the proposed method.

Keywords: Polarimetry, Polarization, Nonuniformly polarized light

2010 MSC: 78-05, 78A55
\end{abstract}

\section{Introduction}

The state of polarization (SoP) of a beam generally changes due to interaction with materials. The analysis of such changes can be used as a non destructive technique to study the optical properties of a given material [1-3]. Usually, it is assumed that the state of polarization is uniform across the transverse section of the light beam. This is the case in most of applications where the use of polarized light is required. However, the state of polarization can vary from point to point across the beam transverse section,

\footnotetext{
* Corresponding author

Email address: jcgsande@ics. upm.es (Juan Carlos González de Sande)
} 
for example, in a periodic way [4-8], with circular symmetry [9-11] or showing more complex structures [12-14].

The SoP of a light beam propagating under paraxial conditions can be represented by means of four real Stokes parameters [1-3]. When such beam impinges onto a specimen, a linear transformation of the Stokes parameters representing the SoP of the beam occurs, and the effect of the sample on the SoP can be described by a $4 \times 4$ real matrix, known as Mueller matrix. Many different techniques have been developed for measuring the Mueller matrix of a sample. In general, this measurement requires the generation of light with four linearly independent SoP and the determination of the SoP of the output light, involving a set of, at least, 16 intensity measurements [2, 3]. Generally, uniformly polarized light across the transverse section is used as input light on the sample. In common configurations for Mueller matrix polarimetry, as dual rotating retarder or dual variable retarder Mueller polarimeters, both the SoP of the input beam and the polarization state analyzer are changed sequentially in time. Afterwards, the analysis of the time varying detector signal permits to extract the Mueller matrix elements [2, 3, 15].

Recently, the use of non uniformly polarized beams has been proposed in order to simultaneously obtain a spatial distribution of SoP's at the entrance of the sample, instead of a time sequence of SoP's [16-19]. In this case, image analysis of the beam intensity after a polarization state analyzer or direct Stokes parameters measurement at different points of the transverse section of the beam can be performed to obtain the sample's Mueller matrix [18, 19].

In general, the SoP distribution across the transverse section of a nonuniformly polarized beam changes under free space propagation and, in many cases, it is not easy to study how it evolves [6, 7, 20]. This problem could be overcome by using nonuniformly polarized beams with an invariant polarization pattern in propagation, as is the case for circularly symmetric spirally polarized beams [9]. It is important to note that, although the polarization state map of a spirally polarized beam changes after passing through a sample, the output SoP map remains invariant in propagation if the sample is homogeneous, linear and deterministic [18].

In the present work, the use of a particular case of spirally polarized beam, an az- 
imuthally polarized beam (APB) [9, 11, 21], is proposed to measure the Mueller matrix of homogeneous linear deterministic samples. The use of APB presents some advantages. All possible linearly polarized states are generated at once and the polarization pattern remains invariant in propagation, so the sample can be placed at any plane along the input beam. Moreover, the SoP map at the output of homogeneous, linear and deterministic samples remains invariant in propagation, so the polarization analyzer of the output beam can be positioned at any plane beyond the sample.

Spirally polarized beams do not contain circular or elliptical SoP's in their cross section. However, the three probing method [22] can be used for measuring $4 \times 3$ elements of the sample's Mueller matrix. On the other hand, an adapted commercial

light polarimeter can be used to measure the Stokes parameters at three different points across the transverse section of the output beam. Then, a partial Mueller matrix of the sample can be obtained in an easy and fast way. After that, taking into account that the Mueller matrix elements for the case of linear and deterministic samples [23] must satisfy a series of relations [24], the complete Mueller matrix of the specimen under study can be recovered.

The structrure of this paper is as follows. The theoretical bases of the measurement method are presented in Section 2, the experimental implementation of the proposed method and the obtained results for several testing samples are described in Section 3 , main findings of this work are summarized in Section 4

\section{Basic concepts}

The SoP of a paraxial beam propagating along a given direction, say $z$, can be described by the real Stokes parameters, that are measurable quantities [1-3, 25]. Although it is usual to deal with uniformly polarized light, in general, the Stokes parameters are functions of the position vector $\mathbf{r}=(r, \theta)$ in the transverse plane of the beam. 
They can be arranged in a four dimensional vector as

$$
\mathbf{S}(r, \theta)=\left[\begin{array}{c}
S_{0}(r, \theta) \\
S_{1}(r, \theta) \\
S_{2}(r, \theta) \\
S_{3}(r, \theta)
\end{array}\right],
$$

where $S_{0}(r, \theta)$ is the intensity of the beam at the point $\mathbf{r}, S_{1}(r, \theta)$ represents the difference between the linearly polarized light with azimuth 0 and $\pi / 2$, respectively, $S_{2}(r, \theta)$ is the difference between the amount of light linearly polarized at $\pi / 4$ and $-\pi / 4$, and finally, $S_{3}(r, \theta)$ is analogous to $S_{2}(r, \theta)$ but considering right and left circular polarized intensity at such point.

In the following, transmission Mueller polarimetry will be considered, although similar results could be obtained for reflection Mueller polarimetry if the effects of the change in the direction of propagation are taken into account. The relation between the output light Stokes vector, $\mathbf{S}^{\text {out }}$ and the input Stokes vector, $\mathbf{S}^{\text {in }}$, can be described by

$$
\mathbf{S}^{\text {out }}(r, \theta)=\widehat{M} \mathbf{S}^{\text {in }}(r, \theta)
$$

where

$$
\widehat{M}=\left(\begin{array}{cccc}
m_{00} & m_{01} & m_{02} & m_{03} \\
m_{10} & m_{11} & m_{12} & m_{13} \\
m_{20} & m_{21} & m_{22} & m_{23} \\
m_{30} & m_{31} & m_{32} & m_{33}
\end{array}\right)
$$

is the $4 \times 4$ Mueller matrix that accounts for changes produced by the interacting object in the SoP of the input light.

The sample's Mueller matrix elements, $m_{i j}, i, j=0,1,2,3$, can be obtained by performing 16 independent intensity measurements. Commonly, a polarization state generator is used to produce, at least, four SoP's whose Stokes vectors are linearly independent. Additionally, the projection of the output light onto, at least, four polarization states with linearly independent Stokes vector are measured for obtaining the complete Mueller matrix [1-3]. 
The proposed method can be equally implemented with any spirally polarized beam. In this work, for simplicity, it will be shown how this method works for the particular case of APBs. They are nonuniformly totally polarized beams whose electric field vector at each point is directed along the azimuthal direction. For these beams, the Stokes vector is given by [9,21]

$$
\mathbf{S}_{A P B}=I(r)\left[\begin{array}{c}
1 \\
-\cos 2 \theta \\
-\sin 2 \theta \\
0
\end{array}\right],
$$

where $I(r, \theta)$ is the intensity of the incident beam, which must be zero at the center because the polarization is not defined at that point.

By measuring the SoP of the input beam at three different points (three different $\theta$ angles) of its cross section and measuring the intensity of the output beam (first Stokes parameter $\left.S_{0}^{\text {out }}(r, \theta)\right)$ at the same positions, the following set of linear equations can be written

$$
\left[\begin{array}{c}
S_{0}^{\text {out }}\left(r_{0}, \theta_{0}\right) \\
S_{0}^{\text {out }}\left(r_{1}, \theta_{1}\right) \\
S_{0}^{\text {out }}\left(r_{2}, \theta_{2}\right)
\end{array}\right]=\widehat{W}\left[\begin{array}{c}
m_{00} \\
m_{01} \\
m_{02}
\end{array}\right]
$$

where $\widehat{W}$ is the polarimetric measurement matrix given by

$$
\widehat{W}=\left[\begin{array}{lll}
S_{0}^{i n}\left(r_{0}, \theta_{0}\right) & S_{1}^{i n}\left(r_{0}, \theta_{0}\right) & S_{2}^{\text {in }}\left(r_{0}, \theta_{0}\right) \\
S_{0}^{i n}\left(r_{1}, \theta_{1}\right) & S_{1}^{\text {in }}\left(r_{1}, \theta_{1}\right) & S_{2}^{\text {in }}\left(r_{1}, \theta_{1}\right) \\
S_{0}^{\text {in }}\left(r_{2}, \theta_{2}\right) & S_{1}^{\text {in }}\left(r_{2}, \theta_{2}\right) & S_{2}^{\text {in }}\left(r_{2}, \theta_{2}\right)
\end{array}\right] .
$$

Note that $S_{3}^{i n}(r, \theta)=0$ for any point at the cross section of an APB.

By properly selecting three different points $\left(r_{k}, \theta_{k}\right)$, in such a way that the input polarization states are represented by three linearly independent Stokes vector, the elements $m_{0 j}$ with $j=0,1,2$ can be obtained by inverting Eq. (5).

In a similar way, if the second, third, and forth Stokes parameters of the output beam $\left(S_{1}^{\text {out }}, S_{2}^{\text {out }}\right.$, and $S_{3}^{\text {out }}$, respectively) are measured at the same points of the transverse section of the beam, the elements $m_{1 j}, m_{2 j}$, and $m_{3 j}$ respectively, can be obtained from 


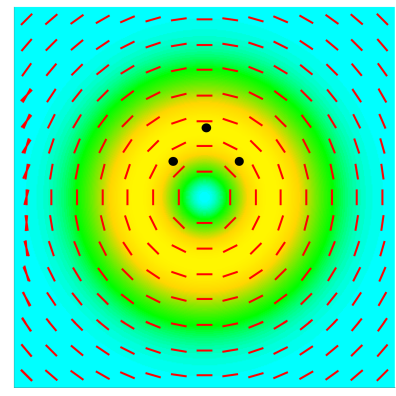

Figure 1: Intensity and polarization pattern for an azimuthally polarized beam. Black dots are an example of three optimum points for measuring the Stokes vector of the input and output beam.

the following equation:

$$
\left[\begin{array}{c}
S_{i}^{\text {out }}\left(r_{0}, \theta_{0}\right) \\
S_{i}^{\text {out }}\left(r_{1}, \theta_{1}\right) \\
S_{i}^{\text {out }}\left(r_{2}, \theta_{2}\right)
\end{array}\right]=\widehat{W}\left[\begin{array}{c}
m_{i 0} \\
m_{i 1} \\
m_{i 2}
\end{array}\right]
$$

where $i=1,2,3$. It must be noted that the polarimetric measurement matrix $\widehat{W}$ is the same for obtaining the first three columns of the Mueller matrix.

Minimization of the condition number of the polarimetric measurement matrix gives the optimum positions for measuring the output beam Stokes parameters [22, 26]. An example of APB is shown in Fig. 11, where the intensity across a transverse section is represented in scale color. The SoP at several points is indicated through the corresponding polarization ellipse (segments for an APB). It can be observed that the SoP is linear everywhere and that the azimuth is perpendicular to the radial direction. The optimum positions for measuring the output beam Stokes parameters when using input linear polarization correspond to points where the input linear polarization states have azimuths equally spaced by $\pi / 3[22]$ and where the intensity of the input beam is close to its maximum, as for example, the black dots shown in Fig. 1 .

Hovenier [24] found up to 30 different relations holding for Mueller matrix elements of linear deterministic samples [23]. More precisely, there are 12 quantities that must vanish, involving products of any two chosen rows or columns, and are of the 
form [24]

$$
\begin{array}{ccc}
\alpha_{1} & = & m_{00} m_{10}-m_{01} m_{11}-m_{02} m_{12}-m_{03} m_{13} ; \\
\alpha_{2} & = & m_{00} m_{20}-m_{01} m_{21}-m_{02} m_{22}-m_{03} m_{23} \\
\vdots & \vdots & \vdots \\
\alpha_{12} & = & m_{02} m_{03}-m_{12} m_{13}-m_{22} m_{23}-m_{32} m_{33}
\end{array}
$$

There are 18 additional quantities, which involve sums or differences of subdeterminants of the Mueller matrix, that have to vanish as well. They can be written in the form [24]

$$
\begin{array}{ccc}
\beta_{1} & = & m_{00} m_{11}-m_{10} m_{01}-m_{22} m_{33}+m_{23} m_{32} ; \\
\beta_{2} & = & m_{00} m_{12}-m_{10} m_{02}+m_{21} m_{33}-m_{23} m_{31} \\
\vdots & \vdots & \vdots \\
\beta_{18} & = & m_{10} m_{21}-m_{11} m_{20}+m_{02} m_{33}-m_{03} m_{32} .
\end{array}
$$

Each quantity $\alpha_{p}$ (with $p=1,2, \ldots, 12$ ) or $\beta_{q}$ (with $q=1,2, \ldots, 18$ ) in Eqs. 8) and 9 involves a certain number of elements belonging to the last column of the complete Mueller matrix. Such number will be denoted by $\gamma_{p}$ or $\gamma_{q}^{\prime}$, respectively. Then the following cost function can be defined:

$$
C=\sum_{p=1}^{12} \alpha_{p}^{2} / \gamma_{p}+\sum_{q=1}^{18} \beta_{q}^{2} / \gamma_{q}^{\prime}
$$

By minimizing the cost function given in Eq. (10), the last column of the Mueller matrix can be obtained if we deal with homogeneous linear deterministic samples.

\section{Application of the method and experimental results}

The proposed method has been experimentally implemented and several testing measurements have been performed with different samples in order to prove its validity. An APB has been synthesized by means of an Arcoptix crystal polarization converter $(P C)$ as shown in the set up of Fig. 2. The light source is a He-Ne laser stabilized in intensity and frequency. The incident state of polarization on the $P C$, which must be linear and parallel to the $P C$ axis in order for the $P C$ to work properly [21, 27], is selected by means of the linear polarizer $P$. The transmission axis of this linear polarizer is along $y$ axis. A microscope objective, $M O_{1}$, and a lens, $L_{1}$, are used to 


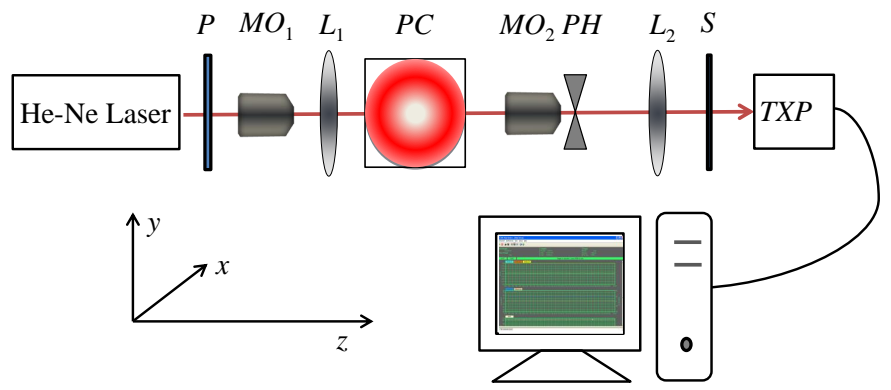

Figure 2: Proposed experimental setup. $P$ : linear polarizer; $M O$ : microscope objectives; $L$ : lenses; $P C$ : polarization converter; $P H$ : Pinhole; $S$ : sample; $T X P$ : light polarimeter.

expand the beam impinging onto the $P C$. The beam after the $P C$ is spatially filtered by means of another microscope objective and a $25 \mu \mathrm{m}$ diameter pinhole $\left(\mathrm{MO}_{2}\right.$ and $\mathrm{PH}$, respectively). The resulting beam is collimated with the convergent lens $L_{2}$ and then impinges on the sample $S$.

A commercial light measuring polarimeter (Thorlabs TXP polarimeter) has been used to analyze the propagated light before and after the sample. This light polarimeter is mounted on a $X Y$-micropositioner stage in order to precisely select the points where to measure the Stokes parameters of the light impinging on the sample and after it. A $500 \mu \mathrm{m}$ diameter pinhole was placed at the entrance of the polarimeter in order to select an area, small enough, where the polarization state can be considered as nearly uniform.

The Stokes parameters of the input beam are measured at three different points. The latter are selected in such a way that their intensities are close to the maximum and the azimuth angles of their polarization states are equally spaced by $\pi / 3$ (see Fig. 1 and experimentally measured Stokes parameters of the beam after lens $L_{2}$ in Tables 1 to 5 .

Three different samples, a quarter-wave plate (QWP), a half-wave plate (HWP), and a Glan-Thompson linear polarizer (LP) have been used as homogeneous, linear and deterministic test samples. Two different orientations, 0 and $\pi / 6$ relative to the $x$ axis, have been selected for the fast axis of the phase plates. The Stokes parameters of the output beam are measured by means of the modified $T X P$ polarimeter at the same three points as those where the input beam has been previously characterized. 
Table 1: Measured Stokes vectors of the input and output beam, normalized to the maximum input intensity, at three different positions for the case of a QWP at 0 [19].

\begin{tabular}{|c|c|c|c|c|c|c|}
\hline Position & & $\left.r_{0}, \theta_{0}\right)$ & $\left(r_{1}\right.$ & $0+\pi / 3)$ & & $\left.\theta_{0}-\pi / 3\right)$ \\
\hline Input beam & $\mathbf{S}^{i n}=$ & $\begin{array}{c}1.0000 \\
-0.9367 \\
-0.0048 \\
-0.0188\end{array}$ & $\mathbf{S}^{i n}=$ & $\begin{array}{l}0.9014 \\
0.4422 \\
0.7676 \\
0.0565\end{array}$ & $\mathbf{S}^{i n}=$ & $\begin{array}{c}0.8280 \\
0.4062 \\
-0.7040 \\
-0.0265\end{array}$ \\
\hline Output beam & $\mathbf{S}^{\text {out }}=$ & $\begin{array}{c}0.9841 \\
-0.9504 \\
0.0011 \\
0.0477\end{array}$ & $\mathbf{S}^{\text {out }}=$ & $\begin{array}{c}0.9207 \\
0.4345 \\
0.0020 \\
-0.8036\end{array}$ & $\mathbf{S}^{\text {out }}=$ & $\begin{array}{c}0.8703 \\
0.4430 \\
-0.0019 \\
0.7321\end{array}$ \\
\hline
\end{tabular}

Tables 1 and 2 shows the measured Stokes vectors for the input beam at three different points, $\left(r_{k}, \theta_{k}\right)$ with $k=0,1,2$, and the corresponding Stokes vectors of the output beam after a QWP with its fast axis along $x$ direction and at $\pi / 6$ radians, respectively. From the values of $\mathbf{S}^{\text {in }}$ at each point, it can be easily checked that the degree of polarization is close to unity (the light is highly polarized), the ellipticity of the ellipse of polarization is near to zero (the state of polarization is almost linear), and the azimuth is close to zero, $\pi / 3$ and $-\pi / 3$ at positions defined by $\left(r_{0}, \theta_{0}\right),\left(r_{1}, \theta_{0}+\pi / 3\right)$, and $\left(r_{2}, \theta_{0}-\pi / 3\right)$, respectively. By using Eq. (7) with the four measured Stokes parameters $S_{i}^{\text {out }}\left(r_{k}, \theta_{k}\right)$ with $i=0,1,2,3$, at the selected three different points, the first three columns of the sample's Mueller matrix are obtained. When linear deterministic samples are considered, as is the present case, the last column can be recovered by imposing several symmetry constraints that the Mueller matrix elements must satisfy [24], following the procedure described in Section 2.

The Mueller matrices obtained from the measurements in Tables 11 and 2 for the mentioned QWP with its axes at two different angles, 0 and $\pi / 6$ radians relative to the $x$ axis, are shown in a scale color in Fig. 3 . Figure 4 shows the absolute value of the differences between theoretical ideal QWP Mueller matrix elements and the corresponding experimentally obtained ones. As it can be noticed, these differences 
Table 2: Measured Stokes vectors of the input and output beam, normalized to the maximum input intensity, at three different positions for the case of a QWP at $\pi / 6 \mathrm{rad}$ [19].

\begin{tabular}{|c|c|c|c|c|c|c|}
\hline Position & \multicolumn{2}{|c|}{$\left(r_{0}, \theta_{0}\right)$} & \multicolumn{2}{|c|}{$\left(r_{1}, \theta_{0}+\pi / 3\right)$} & \multicolumn{2}{|c|}{$\left(r_{2}, \theta_{0}-\pi / 3\right)$} \\
\hline \multirow{4}{*}{ Input beam } & \multirow{4}{*}{$\mathbf{S}^{\text {in }}=$} & 0.9607 & & 1.0000 & \multirow{4}{*}{$\mathbf{S}^{\text {in }}=$} & 0.9971 \\
\hline & & -0.8818 & & 0.4815 & & 0.4841 \\
\hline & & 0.0003 & & 0.8331 & & -0.8380 \\
\hline & & -0.0201 & & 0.0153 & & -0.0234 \\
\hline \multirow{4}{*}{ Output beam } & \multirow{4}{*}{$\mathbf{S}^{\text {out }}=$} & 0.9508 & \multirow{4}{*}{$\mathbf{S}^{\text {out }}=$} & 0.9767 & \multirow{4}{*}{$\mathbf{S}^{\text {out }}=$} & 0.9736 \\
\hline & & -0.2254 & & 0.4691 & & -0.2355 \\
\hline & & -0.4091 & & 0.8107 & & -0.4193 \\
\hline & & -0.7624 & & -0.0004 & & 0.8265 \\
\hline
\end{tabular}

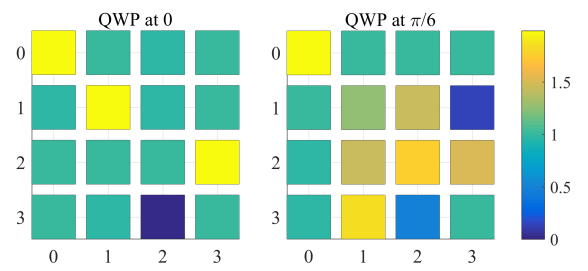

Figure 3: Experimental Mueller matrix for a QWP with its axes at 0 (left) and rotated $\pi / 6$ (right) relative to the $x$ axis [19].
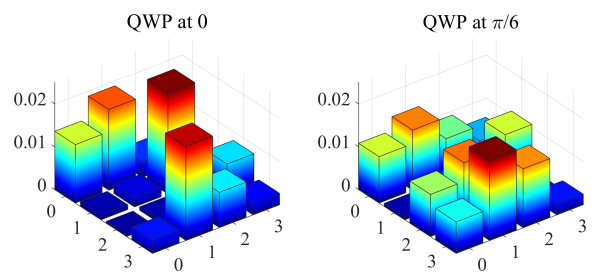

Figure 4: Absolute values of the differences between theoretical and experimental Mueller matrix elements for a QWP with its axes at 0 (left) and rotated $\pi / 6$ (right) relative to the $x$ axis [19].

are very small (less than 0.025 ).

Tables 3 and 4 shows the measured Stokes vectors for the input and output beam after a HWP at three different points. The fast axis has been arbitrarily chosen at 0 and $\pi / 6$ radians relative to $x$ direction, respectively. The procedure described in previous 
Table 3: Measured Stokes vectors of the input and output beam, normalized to the maximum input intensity, at three different positions for the case of a HWP at 0 .

\begin{tabular}{|c|c|c|c|c|c|c|}
\hline \begin{tabular}{|l} 
Position \\
\end{tabular} & \multicolumn{2}{|c|}{$\left(r_{0}, \theta_{0}\right)$} & \multicolumn{2}{|c|}{$\left(r_{1}, \theta_{0}+\pi / 3\right)$} & \multicolumn{2}{|c|}{$\left(r_{2}, \theta_{0}-\pi / 3\right)$} \\
\hline \multirow{4}{*}{ Input beam } & \multirow{4}{*}{$\mathbf{S}^{i n}=$} & 0.9390 & & 0.7250 & & 1.0000 \\
\hline & & 0.9390 & $\sin$ & -0.3460 & $\sin$ & -0.4793 \\
\hline & & -0.0020 & & 0.6014 & & -0.8318 \\
\hline & & 0.0060 & & -0.0071 & & -0.0052 \\
\hline \multirow{4}{*}{ Output beam } & \multirow{4}{*}{$\mathbf{S}^{\text {out }}=$} & 0.9209 & \multirow{4}{*}{$\mathbf{S}^{\text {out }}=$} & 0.7209 & \multirow{4}{*}{$\mathbf{S}^{\text {out }}=$} & 0.9568 \\
\hline & & 0.9228 & & -0.3246 & & -0.4655 \\
\hline & & -0.0010 & & -0.6078 & & 0.8122 \\
\hline & & -0.0140 & & 0.0474 & & 0.0701 \\
\hline
\end{tabular}

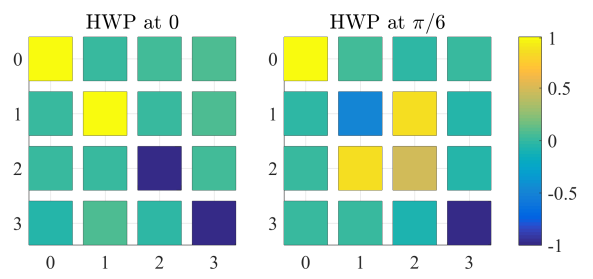

Figure 5: Experimental Mueller matrix for a HWP with its axes at 0 (left) and rotated $\pi / 6$ (right) relative to the $x$ axis.

Section 2 was followed to obtain the corresponding Mueller matrices in each case. The experimental values obtained for these matrices are graphically represented in Fig. 5 . The absolute values of the differences between theoretical and experimental Mueller matrix elements for the case of a HWP with its axes at 0 and rotated $\pi / 6$ radians relative to the $x$ axis are shown in Fig. 6 .

In order to check the performance of the proposed method with diattenuating samples, a linear polarizer is characterized using the setup in Fig. 2 and following the procedure described in Section 2. Table 5 shows the measured Stokes vectors for input and output beam at three appropriately selected points. From these measured values, the Mueller matrix of the linear polarizer is obtained. The resulting matrix, as well as the absolute values of the differences between this experimental Mueller matrix and 
Table 4: Measured Stokes vectors of the input and output beam, normalized to the maximum input intensity, at three different positions for the case of a HWP at $\pi / 6$.

\begin{tabular}{|c|c|c|c|c|c|c|}
\hline Position & \multicolumn{2}{|c|}{$\left(r_{0}, \theta_{0}\right)$} & \multicolumn{2}{|c|}{$\left(r_{1}, \theta_{0}+\pi / 3\right)$} & \multicolumn{2}{|c|}{$\left(r_{2}, \theta_{0}-\pi / 3\right)$} \\
\hline Input beam & $\mathbf{S}^{i n}=$ & $\begin{array}{c}1.0000 \\
-0.9459 \\
0.0038 \\
-0.0183\end{array}$ & $\mathbf{S}^{i n}=$ & $\begin{array}{l}0.7922 \\
0.3875 \\
0.6697 \\
0.0664\end{array}$ & $\mathbf{S}^{i n}=$ & $\begin{array}{c}0.9941 \\
0.4806 \\
-0.8327 \\
-0.0399\end{array}$ \\
\hline Output beam & $\mathbf{S}^{\text {out }}=$ & $\begin{array}{c}0.9440 \\
0.4561 \\
-0.7907 \\
-0.0019\end{array}$ & $\mathbf{S}^{\text {out }}=$ & $\begin{array}{c}0.7873 \\
0.3807 \\
0.6673 \\
-0.0564\end{array}$ & $\mathbf{S}^{\text {out }}=$ & $\begin{array}{c}0.9904 \\
-0.9317 \\
0.0462 \\
0.0606\end{array}$ \\
\hline
\end{tabular}
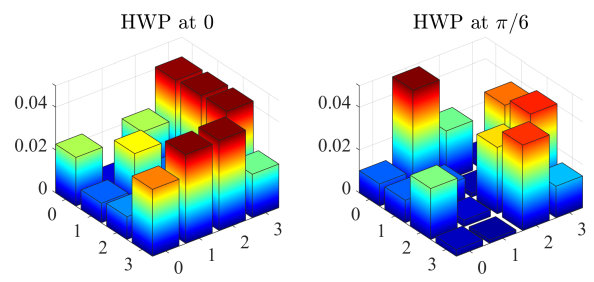

Figure 6: Absolute values of the differences between theoretical and experimental Mueller matrix elements for a HWP with its axes at 0 (left) and rotated $\pi / 6$ (right) relative to the $x$ axis.
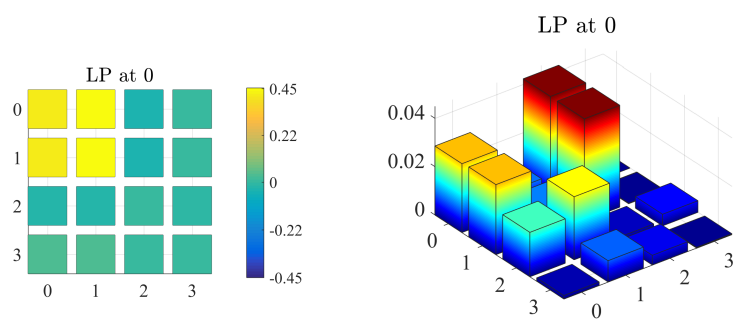

Figure 7: Experimental Mueller matrix (left) and absolute values of the differences between theoretical and experimental Mueller matrix elements (right) for a Glan-Thompson linear polarizer with its transmission axis along the $x$ direction.

the theoretical one are shown in Fig. 7.

From Figs. 4, 6, and 7, it can be seen that the absolute values of the differences 
Table 5: Measured Stokes vectors of the input and output beam, normalized to the maximum input intensity, at three different positions for the case of a HWP at 0 .

\begin{tabular}{|c|c|c|c|c|c|c|}
\hline Position & \multicolumn{2}{|c|}{$\left(r_{0}, \theta_{0}\right)$} & \multicolumn{2}{|c|}{$\left(r_{1}, \theta_{0}+\pi / 3\right)$} & \multicolumn{2}{|c|}{$\left(r_{2}, \theta_{0}-\pi / 3\right)$} \\
\hline \multirow{4}{*}{ Input beam } & \multirow{4}{*}{$\mathbf{S}^{i n}=$} & 0.9349 & & 1.0000 & \multirow{4}{*}{$\mathbf{S}^{i n}=$} & 0.0932 \\
\hline & & 0.9348 & $\sin$ & -0.5029 & & -0.0467 \\
\hline & & -0.0037 & & -0.8643 & & 0.0803 \\
\hline & & -0.0046 & & 0.0049 & & 0.0068 \\
\hline \multirow{4}{*}{ Output beam } & \multirow{4}{*}{$\mathbf{S}^{\text {out }}=$} & 0.8059 & \multirow{4}{*}{$\mathbf{S}^{\text {out }}=$} & 0.2366 & \multirow{4}{*}{$\mathbf{S}^{\text {out }}=$} & 0.0153 \\
\hline & & 0.8047 & & 0.2364 & & 0.0153 \\
\hline & & -0.0416 & & -0.0072 & & -0.0003 \\
\hline & & -0.0096 & & 0.0064 & & -0.0000 \\
\hline
\end{tabular}

between the experimentally obtained Mueller matrix and the theoretical Mueller matrix corresponding to each sample are below 0.05 for all cases. These differences are typically around 0.02 or less for most of the Mueller matrix elements for all studied samples. These values are comparable to the maximum error reported in the Mueller matrix elements measurements by using the dual-rotating retarder method $( \pm 0.034$ [28]), by using variable retarders and rotators together with the analysis of 16 images $( \pm 0.035[29])$ or by using a universal $\mathrm{SU}(2)$ polarization gadget $( \pm 0.02$ [30]). Bueno reported lower error limits in the determination of the Mueller matrix elements when using a polarimetric method based on liquid crystal variable retarders $( \pm 0.014$ [31] $)$. Other authors have proposed the measurement of the Stokes parameters (as the approach used in the present work) for obtaining the Mueller matrix, but reported larger errors $( \pm 0.098$ [32] $)$ than those obtained in the present work.

\section{Conclusions}

In this work, the use of azimuthally polarized beams is proposed to determine the Mueller matrix of homogeneous linear deterministic specimens. The proposed technique is theoretically described, implemented and experimentally tested. An azimuthally polarized beam is used as a simultaneous polarization state generator: all 
possible linearly polarized states of polarization are generated at once. The polarization pattern for this kind of beams is invariant in propagation. Moreover, the polarization pattern obtained after a linear deterministic sample when an azimuthally polarized beam impinges on it, is also invariant. Then, the sample and the polarization state analyzer, can be placed at any plane along the beam. The proposed method is simple and fast: only a commercial Stokes polarimeter with a pinhole attached at its entrance is used to determine the Stokes vector of the input and output beam at three different locations. The best choice of such positions is at the distance from the beam axis where the intensity is maximum and equally spaced by $\pi / 3$ angles. From these measurements, a $4 \times 3$ submatrix of the sample's Mueller matrix is obtained. By imposing the symmetry constraints that the elements of Mueller matrices should satisfy in the case of linear deterministic samples, the last column can be obtained. Experimental results for several samples confirm the validity of the proposed Mueller matrix polarimetry method.

\section{ACKNOWLEDGEMENTS}

This work has been supported by Spanish Ministerio de Economía y Competitividad under projects FIS2013-46475 and FIS2016-75147. 


\section{References}

1.Goldstein DH. Polarized Light. Second (revised and expanded) ed.; Marcel Dekker, Inc.; 2003. ISBN 082474053X.

2.Chipman RA. Polarimetry; vol. I; chap. 15. McGraw-Hill Companies; third ed. ISBN 9780071598890; 2010:1 - 46.

3.Azzam RMA. Stokes-vector and Mueller-matrix polarimetry [Invited]. J Opt Soc Am A 2016;33(7):1396-408. URL: http://josaa.osa.org/abstract.cfm?URI= josaa-33-7-1396 doi $10.1364 /$ JOSAA.33.001396

4.Piquero G, Borghi R, Santarsiero M. Gaussian Schell-model beams propagating through polarization gratings. J Opt Soc Am A 2001;18(6):1399-405. URL: http://josaa.osa.org/ abstract.cfm?URI=josaa-18-6-1399 doi $10.1364 /$ JOSAA.18.001399.

5.Tervo J, Turunen J. Transverse and longitudinal periodicities in fields produced by polarization gratings. Optics Communications 2001;190(16):51 -7. URL: http:// WWW.sciencedirect.com/science/article/pii/S0030401801010938 doi/http:// dx.doi.org/10.1016/S0030-4018(01)01093-8.

6.de Sande JCG, Santarsiero M, Piquero G, Gori F. Longitudinal polarization periodicity of unpolarized light passing through a double wedge depolarizer. Opt Express 2012;20(25):2734860. URL: http://www.opticsexpress.org/abstract.cfm?URI=oe-20-25-27348. doi $10.1364 / 0 \mathrm{E} .20 .027348$

7.Santarsiero M, de Sande JCG, Piquero G, Gori F. Coherencepolarization properties of fields radiated from transversely periodic electromagnetic sources. Journal of Optics 2013;15(5):055701. URL:http://stacks.iop.org/2040-8986/15/i=5/a=055701

8.de Sande JCG, Piquero G, Santarsiero M, Gori F. Partially coherent electromagnetic beams propagating through double-wedge depolarizers. Journal of Optics 2014;16(3):035708. URL:http://stacks.iop.org/2040-8986/16/i=3/a=035708

9.Gori F. Polarization basis for vortex beams. J Opt Soc Am A 2001;18(7):1612-7. URL: http://josaa.osa.org/abstract.cfm?URI=josaa-18-7-1612 doi:10.1364/ JOSAA.18.001612

10.Tervo J. Azimuthal polarization and partial coherence. J Opt Soc Am A 2003;20(10):197480. URL: http://josaa.osa.org/abstract.cfm?URI=josaa-20-10-1974, doi 10 . $1364 /$ JOSAA.20.001974. 
11.Zhan Q. Cylindrical vector beams: from mathematical concepts to applications. Adv Opt Photon 2009;1(1):1-57. URL: http://aop.osa.org/abstract.cfm?URI=aop-1-1-1 doi 10 . $1364 / \mathrm{AOP} .1 .000001$

12.Piquero G, Vargas-Balbuena J. Non-uniformly polarized beams across their transverse profiles: an introductory study for undergraduate optics courses. European Journal of Physics 2004;25(6):793. URL:http://stacks.iop.org/0143-0807/25/i=6/a=011

13.Beckley AM, Brown TG, Alonso MA. Full Poincaré beams. Opt Express 2010;18(10):1077785. URL: http://www.opticsexpress.org/abstract.cfm?URI=oe-18-10-10777. doi $10.1364 / 0 \mathrm{E} .18 .010777$

14.Jones JA, D’Addario AJ, Galvez EJ. Undergraduate laboratory on polarization using poincaré beams. In: Frontiers in Optics 2016. Optical Society of America; 2016:JW4A.66. URL: http://www.osapublishing.org/abstract.cfm?URI= FiO-2016-JW4A.66 doi $10.1364 /$ FIO.2016.JW4A.66

15.Twietmeyer KM, Chipman RA. Optimization of Mueller matrix polarimeters in the presence of error sources. Opt Express 2008;16(15):11589-603. URL: http://www . opticsexpress. org/abstract. cfm?URI=oe-16-15-11589 doi $10.1364 /$ OE.16.011589

16.Tripathi S, Toussaint KC. Rapid Mueller matrix polarimetry based on parallelized polarization state generation and detection. Opt Express 2009;17(24):21396-407. URL: http: //www.opticsexpress.org/abstract.cfm?URI=oe-17-24-21396, doi $10.1364 / 0 \mathrm{E}$. 17.021396

17.Kenny F, Rodríguez O, Lara D, Dainty C. Vectorial polarimeter using an inhomogeneous polarization state generator. In: Frontiers in Optics 2011/Laser Science XXVII. Optical Society of America; 2011:FThQ5. URL: http://www.osapublishing.org/abstract.cfm? URI=FiO-2011-FThQ5 doi 10.1364 /FIO.2011.FThQ5

18.de Sande JCG, Santarsiero M, Piquero G. Spirally polarized beams for polarimetry measurements of deterministic and homogeneous samples. Optics and Lasers in Engineering 2017;91:97 - 105. URL: http://www.sciencedirect.com/science/article/pii/ S0143816616304171 doi http://dx.doi.org/10.1016/j.optlaseng.2016.11.008

19.de Sande JCG, Piquero G, Santarsiero M. Mueller matrix polarimetry by means of azimuthally polarized beams and adapted commercial polarimeter. In: Proceedings of the 5th International Conference on Photonics, Optics and Laser Technology - Volume 1: PHOTOPTICS,. ISBN 978-989-758-223-3; 2017:39-43. 
20.Martínez-Herrero R, Mejías PM. On the propagation of random electromagnetic fields with position-independent stochastic behavior. Optics Communications 2010;283(22):4467

-9. URL: http://www.sciencedirect.com/science/article/pii/ S0030401810004256 doi http://dx.doi.org/10.1016/j.optcom.2010.04.084; electromagnetic Coherence and Polarization.

21.Ramírez-Sánchez V, Piquero G, Santarsiero M. Generation and characterization of spirally polarized fields. Journal of Optics A: Pure and Applied Optics 2009;11(8):085708. URL: http://stacks.iop.org/1464-4258/11/i=8/a=085708

22.Oberemok EA, Savenkov SN. Determination of the polarization characteristics of objects by the method of three probing polarizations. Journal of Applied Spectroscopy 2002;69(1):72-7. URL: http://dx.doi.org/10.1023/A:1015363709617 doi 10 . 1023/A:1015363709617.

23.Simon R. Nondepolarizing systems and degree of polarization. Optics Communications 1990;77(5):349 -54. URL: http://www.sciencedirect.com/science/article/pii/ 003040189090123B doi:http://dx.doi.org/10.1016/0030-4018(90)90123-B

24.Hovenier JW. Structure of a general pure Mueller matrix. Appl Opt 1994;33(36):831824. URL: http://ao.osa.org/abstract.cfm?URI=ao-33-36-8318 doi:10.1364/ A0.33.008318.

25.Born M, Wolf E. Principles of Optics. Sixth (corrected) ed.; Cambridge University Press; 1980. ISBN 0521639212.

26.Zallat J, S A, Stoll MP. Optimal configurations for imaging polarimeters: impact of image noise and systematic errors. Journal of Optics A: Pure and Applied Optics 2006;8(9):807. URL: http://stacks.iop.org/1464-4258/8/i=9/a=015.

27.Ramírez-Sánchez V, Piquero G, Santarsiero M. Synthesis and characterization of partially coherent beams with propagation-invariant transverse polarization pattern. Optics Communications 2010;283(22):4484 -9. URL: http://www.sciencedirect.com/science/article/pii/s0030401810004220 doi http://dx.doi.org/10.1016/j.optcom.2010.04.081, electromagnetic Coherence and Polarization.

28.Goldstein DH. Mueller matrix dual-rotating retarder polarimeter. Appl Opt 1992;31(31):667683. URL: http://ao.osa.org/abstract.cfm?URI=ao-31-31-6676 doi:10.1364/ A0.31.006676. 
29.Justin S. Baba Jung-Rae Chung AHDBDCGLC. Development and calibration of an automated mueller matrix polarization imaging system. Journal of Biomedical Optics 2002;7:7 -. URL: http://dx.doi.org/10.1117/1.1486248, doi $10.1117 / 1.1486248$.

30.Reddy SG, Prabhakar S, Aadhi A, Kumar A, Shah M, Singh RP, Simon R. Measuring the Mueller matrix of an arbitrary optical element with a universal SU(2) polarization gadget. J Opt Soc Am A 2014;31(3):610-5. URL: http: // josaa.osa.org/abstract.cfm?URI= josaa-31-3-610 doi $10.1364 /$ JOSAA.31.000610

31.Bueno JM. Polarimetry using liquid-crystal variable retarders: theory and calibration. Journal of Optics A: Pure and Applied Optics 2000;2(3):216. URL: http://stacks.iop.org/ $1464-4258 / 2 / i=3 / a=308$

32.Dev K, Asundi A. Muellerstokes polarimetric characterization of transmissive liquid crystal spatial light modulator. Optics and Lasers in Engineering 2012;50(4):599 - 607. URL: http: //www.sciencedirect.com/science/article/pii/s0143816611002934, doi http: //dx.doi.org/10.1016/j.optlaseng.2011.10.004; computational Optical Measurement. 